\title{
Reliability and Validity of the Femorotibial Mechanical Axis Angle in Primary Total Knee Arthroplasty: Navigation versus Weight Bearing or Supine Whole Leg Radiographs
}

\author{
Seong Hwan Kim, MD, Yong-Beom Park, MD, Min-Ku Song, MD, Jung-Won Lim, MD, and \\ Han-Jun Lee, MD, PhD \\ Department of Orthopedic Surgery, Chung-Ang University College of Medicine, Seoul, Korea
}

\begin{abstract}
Purpose: To evaluate the reliability and validity of the femorotibial mechanical axis angle from radiographs in the weight bearing (WB) and supine positions compared with navigation-measured values.

Materials and Methods: Sixty-eight cases of navigation-assisted total knee arthroplasty (TKA) were included. The pre- and postoperative whole leg radiographs (WLRs) in WB and supine positions were compared with the initial and final navigation values.

Results: The mean mechanical axis angle from the preoperative WBWLR and navigation were not statistically different $(\mathrm{p}=0.079)$ and were correlated strongly with each other (intraclass correlation [ICC], 0.818). However, on postoperative measurements, although the WBWLR and navigation values were not different ( $\mathrm{p}=0.098$ ), they were not correlated with each other (ICC, 0.093 ). The standard error of measurement was $1.8^{\circ} \pm 3.6^{\circ}$ for the preoperative WBWLR and $2.5^{\circ} \pm 4.8^{\circ}$ for the postoperative WBWLR. The validity that was determined by the Bland-Altman plot was not acceptable for both pre- and postoperative measurements.

Conclusions: The preoperative WBWLR could provide accurate but not precise measurement value of the femorotibial mechanical axis angle for navigation-assisted TKA, and postoperative measurements in navigation were not comparable with radiographic measurements. The lack of agreement was found between the radiographic and navigation measurements of the coronal alignment regardless of pre- or postoperative evaluation although the accuracy was found acceptable.
\end{abstract}

Level of Evidence : Level 4

Keywords: Knee, Arthroplasty, Computer assisted surgery, Radiograph, Reliability, Validity

\section{Introduction}

An effective surgical technique and preoperative planning for

Received May 2, 2018; Revised June 27, 2018;

Accepted August 9, 2018

Correspondence to: Han-Jun Lee, $\mathrm{MD}, \mathrm{PhD}$

Department of Orthopedic Surgery, Chung-Ang University College of

Medicine, 84 Heukseok-ro, Dongjak-gu, Seoul 06974, Korea

Tel: +82-2-2072-2368, Fax: +82-2-764-2718

E-mail: GustinoLHJ@cau.ac.kr

This is an Open Access article distributed under the terms of the Creative Commons Attribution Non-Commercial License (http://creativecommons.org/licenses/by-nc/4.0/) which permits unrestricted non-commercial use, distribution, and reproduction in any medium, provided the original work is properly cited. accurate prosthesis positioning along the mechanical axis in primary total knee arthroplasty (TKA) is crucial to prevention of implant wear and reduction of revision rates ${ }^{1-3)}$.

Many activities of daily living involve weight bearing (WB) knee positions. Therefore, various studies have reported on in vivo WB knee kinematics ${ }^{4-6)}$. However, the effects of $\mathrm{WB}$ on the kinematics of TKA are still unknown. The interest in the kinematics of TKA has recently increased because studies have shown that alteration of knee motion patterns can lead to both abnormal wear of prosthetic components and damage to soft tissue ${ }^{5,7,8)}$. Thus, it is important to evaluate the effects of WB on alignment to determine how to optimize stability in the prosthetic knee. Among many methods that have been introduced to achieve appropriate postoperative alignment, navigation offers 
precise implantation based on anatomical landmarks and kinematic analysis, which improves the precision and consistency of component alignment. The use of an intraoperative navigation system, which has a technical accuracy to within $1^{0^{9-12}}$, allows for objective assessment of the accuracy and reproducibility of radiographic measurements of the TKA components and alignment. However, regardless of the use of navigation, all TKAs in previous studies were performed with the patient in the supine position intraoperatively, whereas evaluation of radiographs of the mechanical axis was usually performed in the WB position. Moreover, in a recent study by Dahabreh et al. ${ }^{13)}$, there was lack of agreement of the alignment between navigation and radiological evaluation. A navigation system is expected to provide accurate measurements of the limb alignment; therefore, more research on the relationship between the navigation and plain radiographic measurements in WB or supine position is needed to clarify the advantages of navigation in measuring the TKA alignment.

The purpose of this study was to evaluate the reliability and validity of the femorotibial mechanical axis angle from radiographs in the WB and supine positions compared to the navigationmeasured femorotibial mechanical axis angle. We hypothesized that WB would affect the femorotibial mechanical axis angle in the preoperative whole leg radiographs (WLRs) and that the femorotibial mechanical axis angle in the supine position would correspond with the navigation-measured angle.

\section{Materials and Methods}

\section{Patients and Methods}

This is a retrospective study on 110 TKAs performed from 2010 to 2011 whose data were collected prospectively. The study was approved by the Institutional Review Board (ID: C2014131 [1327]) and conducted in accordance with the Declaration of Helsinki. WLRs were obtained in the supine and WB positions pre- and postoperatively. To prevent femoral rotation, the ankle was held by an examiner to maintain the patella in a forward position in the supine radiograph and confirmed by the radiograph to ensure the desired position. Navigation-assisted TKAs were performed using a standard medial parapatellar approach and the OrthoPilot (Aesculap, Tuttlingen, Germany) navigation system. The inclusion criteria were varus osteoarthritis patients who underwent TKA using the navigation system. The exclusion criteria were rheumatoid arthritis, severe deformity over $20^{\circ}$ of varus, fixed flexion contracture of more than $30^{\circ}$, valgus knees, revision surgery, inappropriate radiographs, bilateral cases, and body mass index over $30 \mathrm{~kg} / \mathrm{cm}^{2}$ because these criteria could influence the initial setting of the navigation system. After applying the exclusion criteria, 68 TKA patients (11 males and 57 females) were included with a mean age of 68.9 years (range, 64 to 77 years).

After establishing the tracker pin for navigation, the femorotibial mechanical axis angle was measured and recorded by the navigation system as an initial navigation value. Then, medial release was performed to correct the varus deformity, and the posterior cruciate ligament was sacrificed in all patients. An acceptable coronal alignment was determined as within $0^{\circ}$ to $2^{\circ}$ of varus/valgus in the navigation-measured angle after release ${ }^{14)}$. The femoral component rotational angle was defined as the angle of the anteroposterior axis relative to the posterior condylar axis ${ }^{15,16)}$. Then, the femoral cut was made with a femoral cutting block and the tibial cut was made perpendicular to the tibial mechanical axis. After the bone cutting was completed, femoral and tibial trials were inserted, and the varus/valgus angles were reverified with real-time navigation feedback to evaluate collateral ligament balancing. Subsequently, the prostheses were implanted with cement. The measured values were recorded as postoperative navigation values obtained with the knee in neutral position without any stress.

All radiographic measurements were performed on a PACS (General Electric, Chicago, IL, USA) monitor using a mousepoint cursor and an automated computer calculation. Radiological evaluation was based on the pre- and postoperative supinepositioned WLRs (SPWLRs) and WBWLRs (Fig. 1). During surgery, we compared the femorotibial mechanical axis angle of the SPWLR and WBWLR with the navigation-measured femorotibial mechanical axis angle (Fig. 2). After the final component implantation, the navigation-measured femorotibial mechanical axis angle was recorded. At six months after surgery, we compared the angles from the SPWLR and WBWLR with those obtained by navigation. Pre- and postoperative clinical scores, Knee Society Score and Hospital for Special Surgery score were obtained from all patients.

\section{Statistical Analysis}

Statistical analysis was conducted using SPSS ver. 19.0 (IBM Corp., Armonk, NY, USA) and G* power ver. 3.1.5. The primary outcome measure was the difference of three mean values in the preoperative femorotibial mechanical axis angle-the supine, the WB and navigation values. The post hoc power analysis was calculated based on the preoperative mean values and standard deviations for each measurement method. The eta- squared value for three methods was 0.0489 , and the effect size was calculated as 0.2267469 using this eta-squared value with an a error set at $5 \%$. 

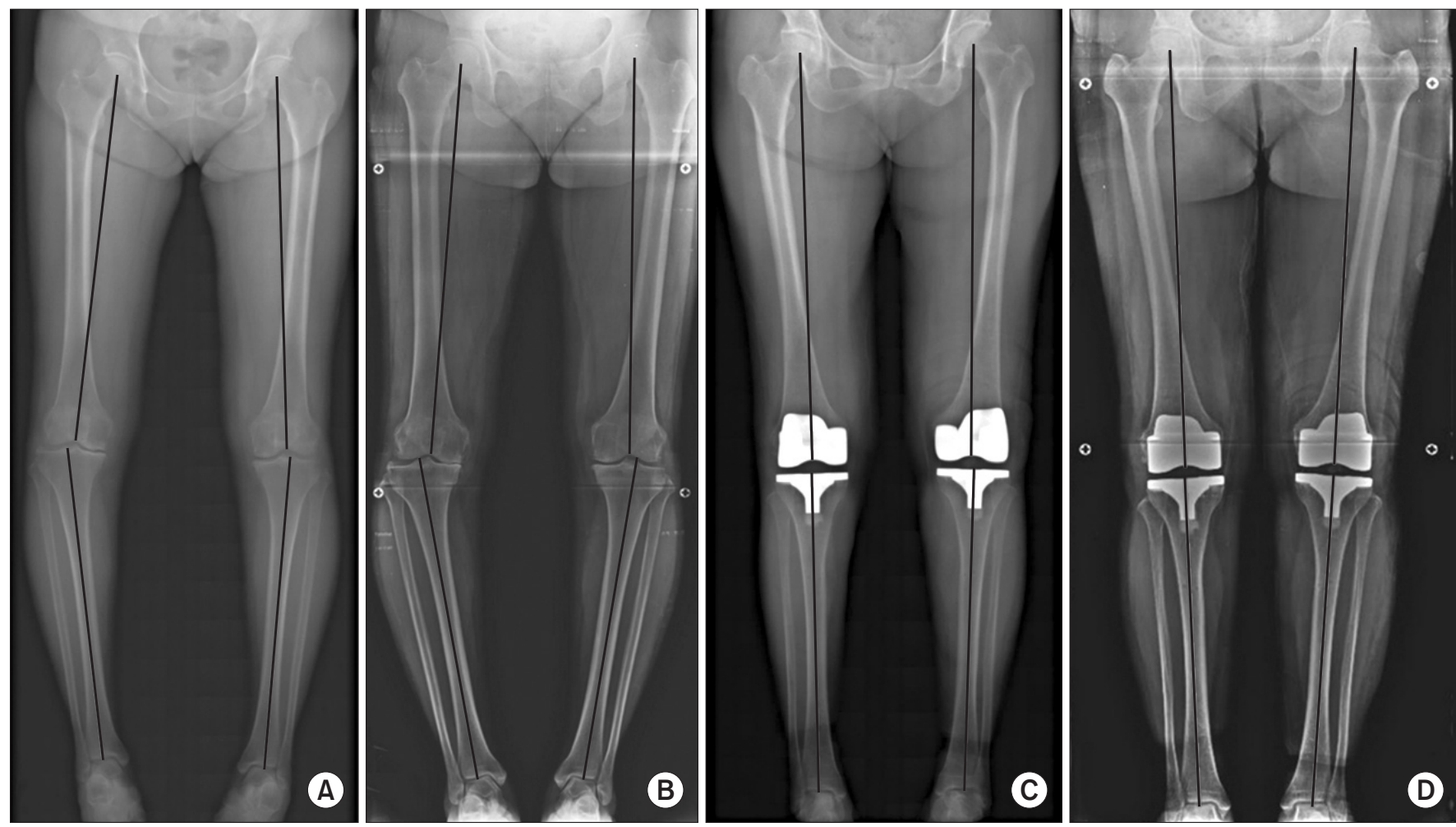

Fig. 1. Preoperative whole leg radiographs (WLRs) in the supine position (A) and weight bearing position (B). Postoperative WLRs in the supine position (C) and weight bearing position (D).
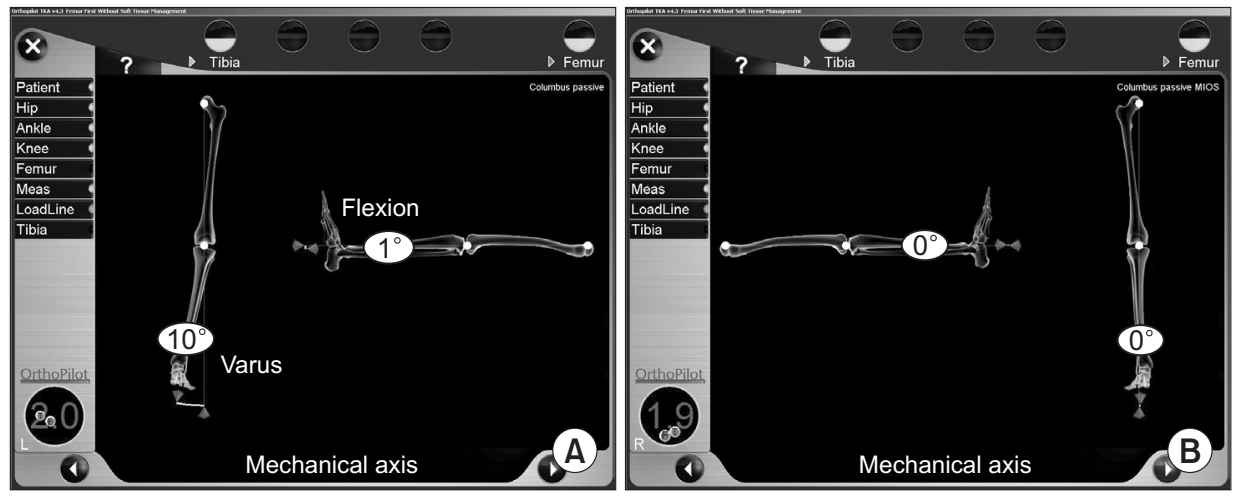

Fig. 2. Navigation values of the mechanical axis during operation (A) and after prosthesis implantation (B).

Based on these calculations, the post hoc power was calculated as 0.9649628 with a $\beta$ error over $80 \%$. The secondary outcome measure was the intraclass correlations (ICCs) for reliability ${ }^{17)}$. With a two-sided $\alpha$ error of $5 \%$ and $\beta$ error of $20 \%$, sample size calculation was performed. The acceptable ICC value for reliability between radiography and navigation was set as 0.9 , and the expected ICC value was set as 0.95 to evaluate the ICC values conservatively. Based on these calculations, the required sample size was 49 for each method; therefore, appropriate power of study was achieved. Two orthopedic surgeons independently measured the pre- and postoperative radiographs with an interval of two weeks between measurements. Test-retest for intra-observer reliability was performed for each surgeon's measurements two weeks after the first measurement. The inter- and intra-observer reliability was calculated with the ICC for consistency.

For comparison of mean values, the data were assessed for normality with the Shapiro-Wilk test. One-way analysis of variance (ANOVA) test with post hoc analysis using Tukey's honestly significant difference test was used to determine the mean differences in the femorotibial mechanical axis angle between the 
supine radiographs, $\mathrm{WB}$ radiographs, and navigation values. The correlations between the femorotibial mechanical axis angles in radiographs (supine and $\mathrm{WB}$ ) and navigation were analysed

Table 1. Analysis of Radiographic and Navigation Measurements of the Lower Limb Alignment

\begin{tabular}{|c|c|c|c|}
\hline Variable & Mean \pm SD & $95 \% \mathrm{CI}$ & p-value ${ }^{a)}$ \\
\hline \multicolumn{4}{|l|}{ Preop value } \\
\hline Preop supine radiograph (A) & $7.7 \pm 6.9$ & 6.5 to 8.9 & $\begin{array}{l}B(p=0.000) \\
C(p=0.000)\end{array}$ \\
\hline Preop WB radiograph (B) & $10.9 \pm 5.2$ & 9.5 to 12.3 & $\begin{array}{l}A(p=0.000) \\
C(p=0.079)\end{array}$ \\
\hline Initial navigation $(\mathrm{C})$ & $10.0 \pm 6.8$ & 8.4 to 11.6 & $\begin{array}{l}A(p=0.000) \\
B(p=0.079)\end{array}$ \\
\hline $\begin{array}{l}\text { Discrepancy between supine } \\
\text { radiograph and navigation (D) }\end{array}$ & $3.3 \pm 2.6$ & 2.7 to 3.9 & $E(p=0.0001)$ \\
\hline $\begin{array}{l}\text { Discrepancy between WB } \\
\text { radiograph and navigation }(\mathrm{E})\end{array}$ & $1.9 \pm 1.3$ & 1.6 to 2.2 & $\mathrm{D}(\mathrm{p}=0.0001)$ \\
\hline \multicolumn{4}{|l|}{ Postop value } \\
\hline Postop supine radiograph & $0.4 \pm 2.6$ & -0.2 to 1.0 & 0.098 \\
\hline Postop WB radiograph & $0.5 \pm 2.9$ & -0.2 to 1.2 & \\
\hline Postop navigation & $0.1 \pm 1.1$ & -0.2 to 0.4 & \\
\hline $\begin{array}{l}\text { Discrepancy between supine } \\
\text { radiograph and navigation }\end{array}$ & $0.6 \pm 1.8$ & 0.2 to 1.0 & 0.46 \\
\hline $\begin{array}{l}\text { Discrepancy between WB } \\
\text { radiograph and navigation }\end{array}$ & $0.8 \pm 1.8$ & 0.4 to 1.2 & \\
\hline \multicolumn{4}{|l|}{ Outlier over $3^{\circ}$ (case) } \\
\hline Postop supine radiograph & & 9 & 0.279 \\
\hline Postop WB radiograph & & 1 & \\
\hline Postop navigation & & 5 & \\
\hline
\end{tabular}

SD: standard deviation, CI: confidence interval, Preop: preoperative, WB: weight bearing, Postop: postoperative.

${ }^{a)} \mathrm{p}<0.05$ was shown between pre- and postoperative values. using Pearson correlation coefficient (PCC). The strength of correlation was indicated by the correlation coefficient $(r)$ as strong $(>0.75)$, moderate $(0.40-0.75)$, or weak $(<0.40)$. Reliability and validity between the navigation and radiographic values were established using ICCs (two way random effect model, absolute agreement), the standard error of measurement (SEM), and 95\% confident interval (CI; 95\% CI of SEM, 1.96×SEM) using the PCC value ${ }^{18)}$. Additionally, Bland-Altman plots and its 95\% limits of agreement (LOA) were used to assess the relationship between the differences and the magnitude between the navigation and radiographic values ${ }^{19)}$. The mean difference in Bland-Altman plot was calculated to evaluate the bias of paired measurements, and the 95\% LOA was calculated to evaluate the degree of agreement of paired measurements. The Shapiro-Wilks test was used to confirm that the measured data followed a normal distribution at 0.05 level of significance, and ICC of 0.70 was selected as the minimally acceptable value for reliability. A chi-square test was used to determine the differences in rates of angles over outliers in postoperative values. Statistical significance was set at $\mathrm{p} \leq 0.05$.

\section{Results}

\section{Differences in Preoperative Radiological Measurements}

The preoperative SPWLR and navigation values were statistically different $(p<0.001)$, but the preoperative WBWLR and navigation values were not statistically different in the one-way ANOVA test with post hoc analysis $(\mathrm{p}=0.079)$ (Table 1$)$. The preoperative SPWLR and navigation measurements for the femorotibial mechanical axis angle were correlated (ICC, 0.743), and the preoperative WBWLR and navigation measurements were more strongly correlated (ICC, 0.818) (Table 2). There was less discrepancy between measurements from the navigation system and those from the preoperative WBWLR than those from the

Table 2. Analysis of the Radiograph-to-Navigation Correlation for Lower Limb Alignment Measurement

\begin{tabular}{|c|c|c|c|c|}
\hline \multirow[b]{2}{*}{ Variable } & \multirow[b]{2}{*}{ Mean $\pm \operatorname{SD}\left({ }^{\circ}\right)$} & \multicolumn{3}{|c|}{ Femorotibial mechanical axis on radiograph: navigation } \\
\hline & & $\begin{array}{c}\text { One-way ANOVA } \\
\text { (p-value) }\end{array}$ & PCC (p-value) & ICC (95\% CI) \\
\hline Initial navigation value & $10.0 \pm 6.8$ & & - & \\
\hline Preop supine radiograph & $7.7 \pm 6.9$ & 0.000 & $0.852(0.000)$ & $0.743(0.513-0.845)$ \\
\hline Preop WB radiograph & $10.9 \pm 5.2$ & 0.079 & $0.876(0.000)$ & $0.818(0.712-0.898)$ \\
\hline Postop navigation value & $0.1 \pm 1.1$ & & & \\
\hline Postop supine radiograph & $0.4 \pm 2.6$ & 0.098 & $0.251(0.04)$ & $0.093(0.034-0.135)$ \\
\hline Postop WB radiograph & $0.5 \pm 2.9$ & & $0.283(0.02)$ & $0.182(0.054-0.246)$ \\
\hline
\end{tabular}

SD: standard deviation, ANOVA: analysis of variance, PCC: Pearson correlation coefficient, ICC: intraclass correlation coefficient, CI: confidence interval, Preop: preoperative, WB: weight bearing, Postop: postoperative. 
preoperative SPWLR. The magnitude of discrepancy between the preoperative radiographs and the navigation system was significantly different between the SPWER and the WBWER $(\mathrm{p}<0.001)$ (Table 1).

Reliability and validity, which was determined by the 95\% LOA and SEM, to detect true differences among the navigation and radiographic values are summarized in Table 3. The SEM and 95\% CI of SEM were found ranging from $2.6^{\circ}$ to $12.8^{\circ}$ for the SPWER and $7.3^{\circ}$ to $14.5^{\circ}$ for the WBWER (Table 3). The Bland-Altman plots revealed upper and lower LOA (95\% LOA) ranging from $-4.7^{\circ}$ to $9.3^{\circ}$ for the SPWER and $-7.6^{\circ}$ to $5.7^{\circ}$ for the WBWER (Fig. 3A and B).

\section{Differences in Postoperative Radiological Measurements}

The mean flexion contracture angle was measured as $2.7^{\circ} \pm 1.2^{\circ}$ with a goniometer at 1 month after surgery, which means pa-

Table 3. Standard Error of Measurement (SEM), 95\% confidence interval (CI) for SEM for Precision, and 95\% Limits of Agreement (LOA)

\begin{tabular}{lrrrr}
\hline \multirow{2}{*}{$\begin{array}{l}\text { Variable } \\
\end{array}$} & Mean \pm SD & \multicolumn{3}{c}{$\begin{array}{c}\text { Overall radiograph: } \\
\text { navigation value }\end{array}$} \\
\cline { 3 - 5 } & & SEM & $\begin{array}{c}95 \% \text { CI } \\
\text { for SEM }\end{array}$ & $\begin{array}{c}95 \% \\
\text { LOA }\end{array}$ \\
\hline Preop supine radiograph $\left(^{\circ}\right)$ & $7.7 \pm 6.9$ & 2.6 & 2.6 to 12.8 & -4.7 to 9.3 \\
Preop WB radiograph $\left(^{\circ}\right)$ & $10.9 \pm 5.2$ & 1.8 & 7.3 to 14.5 & -7.6 to 5.7 \\
Postop supine radiograph $\left(^{\circ}\right)$ & $0.4 \pm 2.6$ & 2.3 & -4.1 to 4.9 & -4.6 to 5.7 \\
Postop WB radiograph $\left(^{\circ}\right)$ & $0.5 \pm 2.9$ & 2.5 & -4.3 to 5.3 & -5.9 to 5.1 \\
\hline
\end{tabular}

SD: standard deviation, Preop: preoperative, WB: weight bearing, Postop: postoperative.

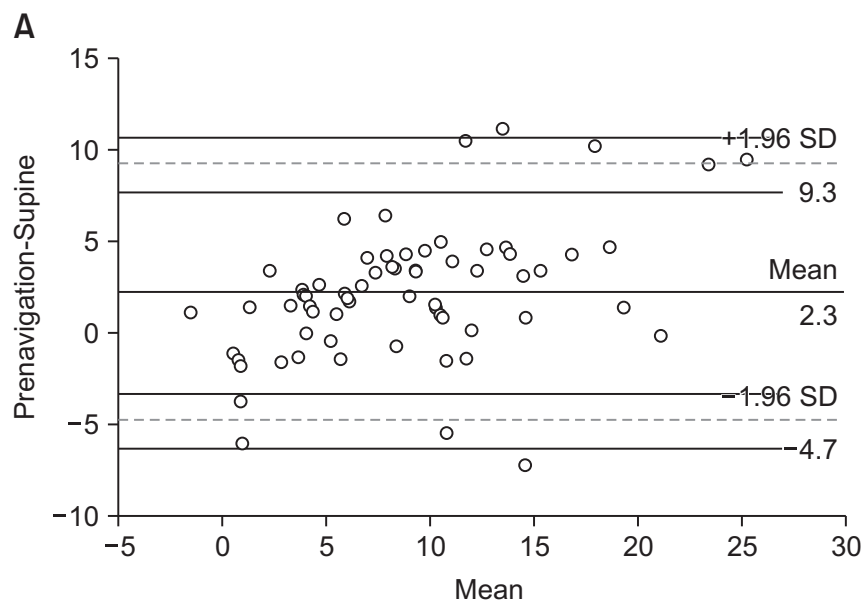

tients achieved nearly full extension. There were no statistical differences in the postoperative SPWLR, WBWLR, and navigation values in one-way ANOVA test $(\mathrm{p}=0.098)$. Moreover, the navigation values were poorly correlated with the SPWLR (ICC, 0.093) and WBWLR (ICC, 0.182) (Table 2). However, the discrepancy between the measurements from the postoperative radiographs and the navigation system did not differ between the SPWLR and WBWLR ( $\mathrm{p}=0.46)$ (Table 1).

Reliability and validity, which was determined by the 95\% LOA and SEM, to detect true differences among the navigation and radiographic values are summarized in Table 3. The SEM and 95\% CI of SEM were found ranging from $-4.1^{\circ}$ to $4.9^{\circ}$ for the SPWLR and $-4.3^{\circ}$ to $5.3^{\circ}$ for the WBWLR (Table 3). The Bland-Altman plots revealed upper and lower LOA (95\% LOA) ranging from $-4.6^{\circ}$ to $5.7^{\circ}$ for the SPWLR and $-5.9^{\circ}$ to $5.1^{\circ}$ for the WBWLR (Fig. 4).

A chi-square test did not reveal any differences in the number of outliers over $3^{\circ}$ for coronal plane alignment among the three measurement methods ( $p>0.05$ ) (Table 1). The measured intraand inter-observer correlation using ICC ranged from 0.893 to 0.978 for all measurements, indicating good to excellent reliability.

\section{Discussion}

The most important finding of this study is that the preoperative femorotibial mechanical axis angle in the WB radiograph correlated more closely with the navigation value than with that in the supine radiograph; thus, we rejected our first hypothesis

\section{B}

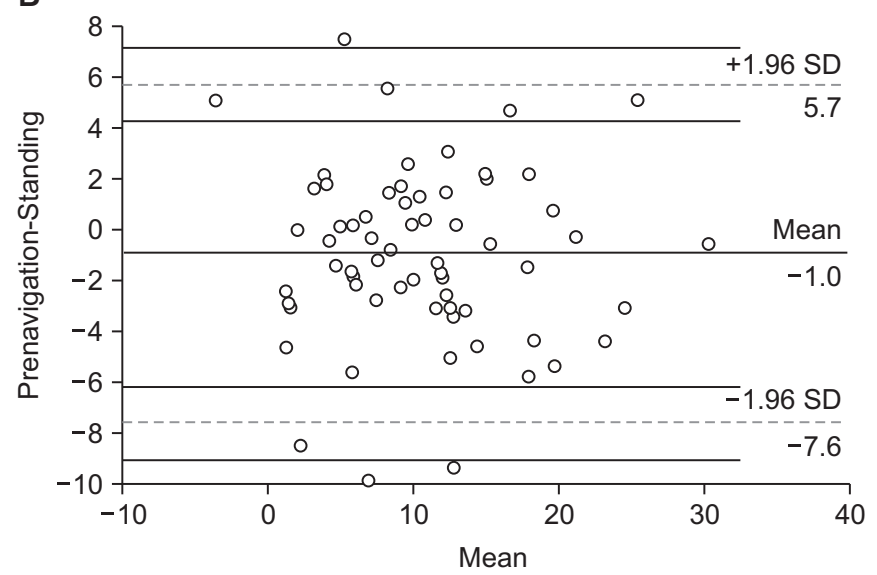

Fig. 3. Bland-Altman plots for agreement of navigation and preoperative measurements. (A) Plot for preoperative supine radiographic and navigation values. (B) Plot for preoperative standing radiographic and navigation values. The Bland-Altman plot showed less discrepancy between weight bearing whole leg radiographs (WLRs) and navigation values, but the range of $95 \%$ limits of agreement was not acceptable for both the supine-positioned WLRs and weight bearing WLRs. SD: standard deviation. 

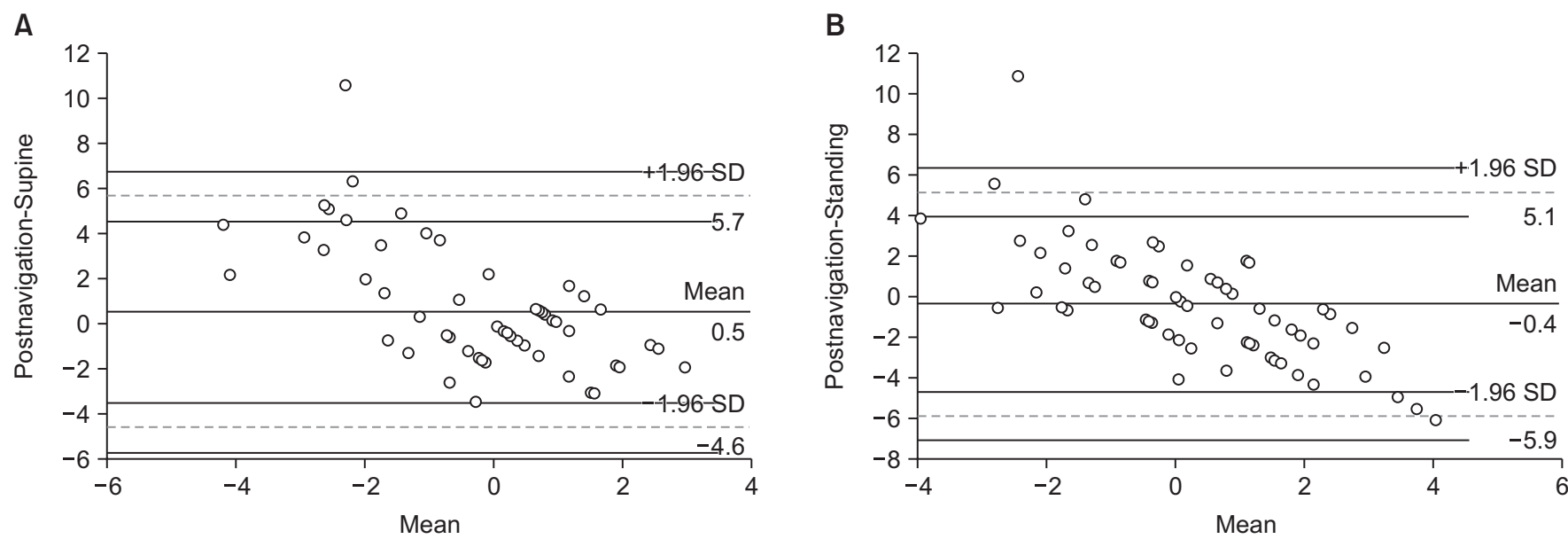

Fig. 4. Bland-Altman plots for agreement between navigation and postoperative measurements. (A) Plot for postoperative supine radiographic values and navigation values. (B) Plot for postoperative standing radiographic values and navigation values. The Bland-Altman plot showed similar discrepancy between the supine/standing radiographic values and navigation values, and the range of 95\% limits of agreement was not acceptable. Moreover, the radiographic values and navigation values showed the proportional error of measurements. SD: standard deviation.

that the navigation value could be more correlated with the supine angle. There was no correlation between the postoperative alignment measured by navigation and radiographic evaluations although postoperative mean values and outliers were not statistically different between the two methods. Moreover, the validity between the radiographic evaluations and the navigation values were found very low in both preoperative and postoperative evaluations.

Precise perioperative measurements of limb alignment are necessary to plan, perform, and evaluate TKA. Under ideal circumstances, standard radiographs are accurate in normal patients with minimal deformity ${ }^{20)}$. However, these conditions are difficult to meet in practice and do not apply to patients with extremitydeforming osteoarthritis. Moreover, deformity can give rise to soft tissue imbalance that can cause changes in the overall alignment under WB.

Most previous studies on TKA have not examined whether WB or non-WB radiographic measurements more strongly correlated with navigation values. The femorotibial mechanical axis angle in navigation is generally recorded in the supine position. Thus, we hypothesized that the navigation values would more likely correlate with the preoperative non-WB radiographic measurements. However, the preoperative WB radiographic measurements more closely correlated with the navigation values. When recording the navigation values, we adjusted the patient's limb manually to detect the probes. During this manipulation, application of axial forces was unavoidable. This lower extremity position may have an influence on deforming force of the knee. Moreover, arthrotomy to locate the navigation probe and to expose the anatomic references was performed before recording the femorotibial mechanical axis angle in navigation, so the true supine condition of preoperative evaluation could not be achieved after soft tissue release. Nevertheless, the femorotibial mechanical axis angle in the WB radiographs strongly correlated with the navigation values preoperatively. Previous studies reported that a trend of greater discrepancy was found between radiography and navigation in patients with larger lower limb deformities ${ }^{11,211}$. Because we excluded severe deformities and severe fixed flexion contracture patients, the correlations between navigation and radiological measurement showed good ICC values (Table 2) regardless of WB or non-WB position, although statistical difference was found between navigation and supine values $(\mathrm{p}=0.000)^{9-12,22}$. Among them, the correlation between the preoperative radiographic measurements and navigation values was found to be 0.885 by Yaffe et al. ${ }^{11)}$. This is consistent with the strong correlations we found for the preoperative radiographic and navigation measurements of the femorotibial mechanical axis angle in the present study.

Another important finding of this study is that there was no correlation between the navigation and radiographic measurements of the postoperative femorotibial mechanical axis angle. The ICC values for correlation in postoperative measurements were very low (Table 2) regardless of WB. However, the changes of less than $1^{\circ}$ in radiographic measurement would exist in the range of precision limits of the navigation measurements ${ }^{9,10,12)}$; the evidence to confirm whether the postoperative correlation analysis is significant or not would be insufficient due to the measured values are all in the measurement error range. Moreover, since the ligamentous imbalance and the deformity were corrected 
after surgery, the effect of WB on alignment would be limited postoperatively. Despite this, the postoperative mean values and outliers were not significantly different between different measurement methods. In brief, if the postoperative navigation values were within the outlier range, then the postoperative femorotibial mechanical axis angle would also be considered within the outlier range. This finding is consistent with the results reported by Yaffe et al. ${ }^{11}$, Willcox et al. ${ }^{21}$, and Choi et al. ${ }^{23)}$ but not with those of Hauschild et al. ${ }^{22}$. Hauschild et al. ${ }^{22)}$ suggested that the radiographic and navigation measurements for TKA would correlate well in both the pre- and postoperative period when the radiographs are delayed. Our results showed worse correlation in postoperative values despite delayed measurement. Thus, controversy still exists about whether postoperative navigation measurements correlate with radiographic values.

Navigation-assisted TKA is known as a highly reliable system with a mean error of less than $1^{0^{9)}}$. However, in this study, the lack of agreement between the navigation value and radiographic values was found. This low reliability was also found in the study of Dahabreh et al. ${ }^{13)}: 20 \%$ of mismatch in coronal alignment was found between the navigation values and radiographic values by CT. According to the Bland-Altman plot for the results of $\mathrm{Da}-$ habreh et al. ${ }^{13)}$, the LOA was found to range from $-5.4^{\circ}$ to $4.7^{\circ}$, similar to our result of postoperative standing value. Although the mean differences in Bland-Altman plot were $-0.4^{\circ}$ in our study and $-0.32^{\circ}$ in Dahabreh's results ${ }^{13)}$, the ranges of LOA were not acceptable in both studies. However, the values of coronal alignment in navigation and radiography were not statistically different, the frequency of outliers was also not different, and the repeatability or reproducibility of the navigation measurement was shown acceptable.

An appreciation of the limitations and potential sources of error inherent to each of the discussed measurement modalities is warranted. First, human error during the process of alignment evaluation or operation is a potential source of measurement variability. For the radiographic measurement, the position of the knee joint could affect the alignment. Second, there were fundamental errors in detecting the navigation probe, such that the true supine femorotibial mechanical axis angle was hard to obtain, especially in patients with deformity. Nevertheless, severe deformities were excluded from this study; further study would be needed in patients with severe deformities to confirm the correlation. Third, the relatively large number of female patients could have affected the measurements. However, due to the exclusion criteria of this study, the study population consisted of patients commonly encountered in clinics, and thus we thought the results are worth to be analysed and reported. Finally, the potential for measurement errors exists because the decimals in radiographic values and navigation values were different. The navigation values were recorded with no decimal point, but the radiographic values were recorded with two decimal values which were rounded to one decimal place for analysis; further studies that address these differences are needed. In spite of these limitations, our study is one of the few comprehensive studies to compare $\mathrm{WB}$ and supine radiographs and navigation and it provides a guide to surgeons on the limitations of the navigation system for TKA.

\section{Conclusions}

The preoperative WBWLR could provide accurate but not precise measurement of the femorotibial mechanical angle for navigation-assisted TKA, and the postoperative measurement with navigation could not be comparable with the radiographic measurements. The lack of agreement was found between the radiographic and navigation measurements in coronal alignment regardless of pre- and postoperative evaluation, although the accuracy was found acceptable.

\section{Conflict of Interest}

No potential conflict of interest relevant to this article was reported.

\section{References}

1. Figgie HE 3rd, Goldberg VM, Figgie MP, Inglis AE, Kelly M, Sobel M. The effect of alignment of the implant on fractures of the patella after condylar total knee arthroplasty. J Bone Joint Surg Am. 1989;71:1031-9.

2. Laskin RS. The classic: total condylar knee replacement in patients who have rheumatoid arthritis: a ten-year follow-up study: 1990. Clin Orthop Relat Res. 2008;466:2589-96.

3. Weng YJ, Hsu RW, Hsu WH. Comparison of computerassisted navigation and conventional instrumentation for bilateral total knee arthroplasty. J Arthroplasty. 2009;24:66873.

4. Stiehl JB, Komistek RD, Dennis DA, Paxson RD, Hoff WA. Fluoroscopic analysis of kinematics after posterior-cruciateretaining knee arthroplasty. J Bone Joint Surg Br. 1995;77: 884-9.

5. Dennis DA, Komistek RD, Mahfouz MR. In vivo fluoroscopic analysis of fixed-bearing total knee replacements. 
Clin Orthop Relat Res. 2003;(410):114-30.

6. Suggs JF, Hanson GR, Park SE, Moynihan AL, Li G. Patient function after a posterior stabilizing total knee arthroplasty: cam-post engagement and knee kinematics. Knee Surg Sports Traumatol Arthrosc. 2008;16:290-6.

7. Moschella D, Blasi A, Leardini A, Ensini A, Catani F. Wear patterns on tibial plateau from varus osteoarthritic knees. Clin Biomech (Bristol, Avon). 2006;21:152-8.

8. Ho FY, Ma HM, Liau JJ, Yeh CR, Huang CH. Mobile-bearing knees reduce rotational asymmetric wear. Clin Orthop Relat Res. 2007;462:143-9.

9. Pitto RP, Graydon AJ, Bradley L, Malak SF, Walker CG, Anderson IA. Accuracy of a computer-assisted navigation system for total knee replacement. J Bone Joint Surg Br. 2006; 88:601-5.

10. Rauh MA, Boyle J, Mihalko WM, Phillips MJ, Bayers-Thering M, Krackow KA. Reliability of measuring long-standing lower extremity radiographs. Orthopedics. 2007;30:299-303.

11. Yaffe MA, Koo SS, Stulberg SD. Radiographic and navigation measurements of TKA limb alignment do not correlate. Clin Orthop Relat Res. 2008;466:2736-44.

12. Lutzner J, Gross AF, Gunther KP, Kirschner S. Reliability of limb alignment measurement for high tibial osteotomy with a navigation system. Eur J Med Res. 2009;14:447-50.

13. Dahabreh Z, Scholes CJ, Giuffre B, Coolican MR, Parker DA. Lack of agreement between computer navigation and post-operative 2-dimensional computed tomography (CT) measurements for component and limb alignment in total knee arthroplasty (TKA). Knee. 2016;23:137-43.

14. Kim SH, Lim JW, Ko YB, Song MG, Lee HJ. Comparison of ultra-congruent mobile- and fixed-bearing navigation-assisted total knee arthroplasty with minimum 5-year followup. Knee Surg Sports Traumatol Arthrosc. 2016;24:34663473.
15. Matsuda S, Miura H, Nagamine R, Mawatari T, Tokunaga M, Nabeyama R, Iwamoto Y. Anatomical analysis of the femoral condyle in normal and osteoarthritic knees. J Orthop Res. 2004;22:104-9.

16. Lustig S, Lavoie F, Selmi TA, Servien E, Neyret P. Relationship between the surgical epicondylar axis and the articular surface of the distal femur: an anatomic study. Knee Surg Sports Traumatol Arthrosc. 2008;16:674-82.

17. Walter SD, Eliasziw M, Donner A. Sample size and optimal designs for reliability studies. Stat Med. 1998;17:101-10.

18. Harvill LM. Standard error of measurement. Educ MeasIssues Pract. 1991;10:33-41.

19. Bland JM, Altman DG. Statistical methods for assessing agreement between two methods of clinical measurement. Lancet. 1986;1:307-10.

20. Cooke TD, Scudamore RA, Bryant JT, Sorbie C, Siu D, Fisher B. A quantitative approach to radiography of the lower limb: principles and applications. J Bone Joint Surg Br. 1991; 73:715-20.

21. Willcox NM, Clarke JV, Smith BR, Deakin AH, Deep K. A comparison of radiological and computer navigation measurements of lower limb coronal alignment before and after total knee replacement. J Bone Joint Surg Br. 2012;94:123440.

22. Hauschild O, Konstantinidis L, Baumann T, Niemeyer P, Suedkamp NP, Helwig P. Correlation of radiographic and navigated measurements of TKA limb alignment: a matter of time? Knee Surg Sports Traumatol Arthrosc. 2010;18:131722.

23. Choi WC, Lee S, An JH, Kim D, Seong SC, Lee MC. Plain radiograph fails to reflect the alignment and advantages of navigation in total knee arthroplasty. J Arthroplasty. 2011; 26:756-64. 